\title{
QUELQUES ASPECTS DES MÉCANISMES NUTRITIONNELS CHEZ L'ISOPODE CYMOTHOIDAE : CERATOTHOA OESTROIDES (RISSO, 1826)
}

\author{
B. ROMESTAND, P. THUET et J.-P. TRILLES
}

RÉSUMÉ. Chez l'Isopode Cymothoidae Ceratothoa oestroides, parasite hématophage de la Bogue (Boops boops; poisson Téléostéen, Sparidae), une étude de certains aspects des mécanismes nutritionnels est envisagée chez les individus en phase sexuelle femelle.

Dans un premier temps, l'étude des variations qualitatives (réplétion de l'hépatopancréas, coloration des différentes parties) et des variations quantitatives (variations pondérales) du tube digestif et de ses annexes permet de noter que l'absorption du sang est étroitement liée aux stades du développement sexuel et du cycle d'intermue. Le schéma général semble le suivant : la prise de nourriture s'effectue avant ou au tout début de la vitellogenèse et après la libération des pulli. L'utilisation des nutriments ingérés et des réserves permet d'assurer la vitellogenèse et le développement des larves intramarsupiales. Dans un deuxième temps, nous avons essayé de rechercher s'il existe un mécanisme déclenchant l'absorption du sang et quelle pourrait être sa nature ? Un tel mécanisme parait effectivement exister ; il semble en partie tributaire d'un facteur stimulant tel que la pression osmotique ou la composition ionique du sang de l'hôte. L'existence de chémorécepteurs est d'ailleurs envisagée ; ces derniers auraient vraisemblablement pour localisation les pièces buccales, la cavité buccale ou peut être même la région œsophagienne des parasites.

\section{Some aspects of nutritional mechanisms in the Isopod Ceratothoa oestroides Risso, 1826 parasite of fishes.}

SUMMARY. Some nutritional mechanisms of the isopod Cymothoidae Ceratothoa oestroides, haematophagic parasite of the teleostean sparid fish Boops boops have been studied in female sexual phase individuals.

The study of qualitative (hepatopancreas repletion, different colouration) and quantitative variations (weight variations-) of digestive tract and its appendages has permitted to observe that blood absorption is close related to sexual developmental stages and intermolt cycle. The general pattern seems as follows: food intake is made prior or just before vitellogenesis and after releasing of "pulli". The use of ingested nutriments and reserves allow vitellogenesis and intramarsupium larval development.

We have also tried to investigate if it does exist a mechanism starting blood absorption and attempted to elucidate its nature. A such mechanism seems indeed to exist, it might have been partially dependent upon a stimulus such as osmotic pressure or ionic composition of host blood. Moreover, chemoreceptors activity is envisaged those could likely occur as bucal appendages, bucal cavity or even the oesophagian region of parasites.

* Centre de Physiologie des Invertébrés, Université des Sciences et Techniques du Languedoc, Place E. Bataillon, F34060 Montpellier Cedex.

Accepté le 27 mars r98r. 


\section{Introduction}

Les Isopodes Cymothoidae (individus mâles et femelles) sont des ectoparasites à l'état adulte de poissons marins ou d'eaux douces. Leur régime alimentaire est normalement exclusivement hématophage (Schioedte, 1868; De Scalzi, 1941; Legrand, 1952 ; Trilles, 1968 ; Monod, 1976 ; Romestand et Trilles, 1976 ; Romestand, 1979) et on observe un certain nombre de modifications du tube digestif en rapport avec ce mode de nutrition particulier. On trouve ainsi chez ces animaux :

- Une bouche ventrale entourée de pièces buccales puissantes et acérées permettant de dilacérer les téguments de l'hôte et de percer les vaisseaux sanguins (Schioedte, 1868 ; De Scalzi, 1941 ; Lombardo, 1975).

- Un œsophage de structure simple mais muni de fibres musculaires radiaires (Ide, 1893 ; Trilles, 1968) réglant ses contractions et ses dilatations.

- Deux glandes latéro-œesophagiennes (fig. 2A) (Gabe, 1960; Fain-Maurel, 1966, Romestand et Trilles, 1976 ; Romestand, 1979), annexées au tube digestif, dont les canaux excréteurs débouchent de part et d'autre de la bouche au niveau des mandibules. Elles sécrètent un principe anticoagulant à activité antithrombique de type héparine (Romestand et Trilles, 1976).

Des travaux effectués sur certains représentants de cette famille de parasites, ont également permis à l'un d'entre nous de mettre en évidence :

- une action hémolysante, surtout de l'hépatopancréas, à un moindre degré de

l'intestin et des diverticules intestinaux postérieurs (Romestand, 1979) ;

- une absorption des substances résultant de la dégradation du sang et plus précisé-

ment de l'hémoglobine (hématéine, hématine et fer), au niveau de l'hépatopancréas

(Romestand, 1979).

Ajoutons qu'au cours des récoltes, on peut noter une très grande variabilité de l'aspect, de la couleur, etc. du tube digestif des Cymothoadiens. Ces observations rejoignent celles effectuées par Trilles en 1968; l'auteur a en effet constaté que les individus présentent des phases cycliques d'alimentation, avec toutefois des modalités différentes pour les mâles et les femelles.

Pour essayer de compléter les résultats précédemment acquis sur l'hématophagie des Cymothoadiens, et afin de préciser certains aspects de leurs mécanismes nutritionnels, nous avons entrepris :

- l'étude des différentes phases de réplétion de l'hépatopancréas, des variations de la coloration (aspects qualitatifs) et des variations pondérales (aspects quantitatifs) des différentes parties du tube digestif en fonction des différents stades de développement sexuel ;

- l'étude du mécanisme (si mécanisme il y a ?) qui déclenche l'ingestion de sang par le parasite.

\section{Matériel biologique et méthodes expérimentales}

L'espèce retenue appartient à la catégorie écologique des Cymothoadiens buccaux (Trilles, 1968) ; il s'agit de Ceratothoa oestroides (Risso, 1826). Ce parasite est récolté 
sur un poisson Téléostéen de la famille des Sparidae, Boops boops (Linnaeus, 1758). Poissons et parasites sont pêchés au large des ports d'Agde et de Sète (Hérault, France). Ramenés au laboratoire, les Cymothoadiens sont mis en élevage dans des bacs en matière plastique contenant 50 litres d'eau de mer filtrée en circuit fermé par des dispositifs pompes et filtres Eheim.

Nos observations ont toujours été effectuées sur du matériel frais, récemment récolté ( 1 jour après la récolte); nous n'avons expérimenté que sur des individus en phase sexuelle femelle. Ils ont été répartis en 6 stades sexuels :

— stade I : individus avant la vitellogenèse

— stade II : individus en vitellogenèse

— stade III $^{1}$ : individus ovigères

— stade IV : individus avec des œufs embryonnés dans le marsupium

- stade V : individus avec des pulli dans le marsupium

— stade VI ${ }^{2}$ : individus ayant mis bas (marsupium vide).

Les dissections ont toujours été réalisées dans l'eau de mer, le tube digestif (6 caecums hépatopancréatiques, intestin et 2 diverticules intestinaux postérieurs ( fig. $2 \mathrm{~A}$ ) étant prélevé sur les individus par électrocoagulation des régions antérieure (œsophagienne) et postérieure (en arrière des diverticules intestinaux).

L'examen des phases de réplétion de l'hépatopancréas, nous a conduit à définir 7 groupes distincts suivant la région du péréion atteinte par l'extrémité distale des caecums entériques. Exemple : groupe 7 : l'extrémité distale de l'hépatopancréas atteint la limite postérieure du $7^{\mathrm{e}}$ péréionite.

Dans le cas des variations pondérales de l'appareil digestif, nous avons rapporté le poids frais du tube digestif (total ou de ses différentes parties) à celui des individus.

Pour l'étude des mécanismes éventuels déclenchant la prise de nourriture, nous avons imaginé le dispositif expérimental suivant : les individus femelles (stade III) sont disposés verticalement dans des bouchons en matière plastique perforés ; ils sont maintenus et fixés par du ciment de dentiste. La partie postérieure portant les lames pléopodiales respiratoires est immergée dans de l'eau de mer ; le céphalon est recouvert de liquide de boisson constituée soit par de l'eau de mer, soit par de l'eau de mer diluée de telle sorte que sa pression osmotique soit égale à celle du sang de poisson ; dans les deux cas, le liquide de boisson est coloré par du bleu de méthylène. Afin d'éviter des remontées d'eau de mer de la partie postérieure vers la région céphalique, nous recouvrons l'eau de mer immergeant les pléopodes de l'animal, d'une couche d'huile de paraffine de $1 \mathrm{~cm}$ environ. Cette précaution est indispensable afin d'obtenir des résultats reproductibles. La durée de l'expérience a été fixée à 4 heures. Une fois celle-ci terminée, les individus sont disséqués et l'observation du tube digestif réalisée sous la loupe binoculaire permet de déterminer s'il y a eu absorption du liquide de boisson. La quantité de liquide absorbé est appréciée en notant l'état de réplétion de l'intestin (longueur et intensité du segment coloré). Elle est évaluée de la manière

I. Entre les stades II et III, se situe une mue qui permet à la femelle d'acquérir ses 5 paires d'oostégites.

2. Après le stade VI, la femelle effectue une mue au cours de laquelle elle perd ses oostégites. 
suivante : 0 , aucune absorption ; 1 , seule le $1 / 4$ antérieur de tube digestif est coloré ; 2 , la moitié antérieure du tube digestif est colorée ; 3 , la totalité du tube digestif est faiblement colorée ; 4 , la totalité du tube digestif est fortement colorée.

Ce type d'expérience a également été réalisé en ajoutant aux deux liquides de boisson de l'EDTA $51 \mathrm{Cr}$ (activité volumique : $1 \mu \mathrm{Ci} / \mathrm{ml})^{1}$. Au bout de 4 heures, la radioactivité du tube digestif disséqué et rincé est mesurée dans un cristal puits en INa.

\section{Résultats}

Aspects qualitatifs : les variations du tube digestif et de la longueur des caecums hépatopancréatiques en fonction des stades sexuels femelles sont indiquées sur la figure $1 A$ et $B$, (tableau $I$ ).

Nous pouvons ainsi noter :

- qu'en ce qui concerne la réplétion de l'hépatopancréas (fig. 1A), il se produit du stade $\mathrm{I}$ au stade $\mathrm{V}$ inclus, un déplacement de pourcentage maximum d'individus vers la droite, ce qui traduit un raccourcissement progressif des caecums entériques ; par la suite (stade VI), le pic est déplacé vers la gauche (groupe 4), ce qui correspond à un passage de sang récemment ingéré par le parasite de l'intestin vers l'hépatopancréas.

- que les différentes parties du tube digestif présentent une évolution particulière de leur coloration ( fig. 1B); ainsi :

- au niveau de l'hépatopancréas, en général à partir du stade III, il se produit un changement de coloration, avec passage du brun foncé au brun clair ; il est en relation avec l'évolution de la digestion du sang. Du stade III au stade VI, la coloration brun clair est de plus en plus marquée et fréquente ( $77 \%$ des individus).

- Aux stades I, III et VI, on trouve un nombre important d'individus présentant un contenu intestinal rougeâtre (sang de poisson récemment ingéré). Des stades I à VI, on observe toujours un pourcentage élevé d'individus présentant un liquide intestinal jaunâtre, des substances de coloration blanchâtre apparaissent de façon plus irrégulière pour les stades I, II, III et VI. Par contre, quel que soit le stade à l'exception du stade IV, des individus à l'intestin vide ne sont rencontrés qu'irrégulièrement.

- Au niveau des diverticules intestinaux postérieurs, il ne nous a pas été possible de trouver un cycle de coloration lié à l'état sexuel des femelles; dans presque tous les $\mathrm{cas}^{2}$, ces organes sont de couleur brun orangé.

\section{Aspects quantitatifs :}

En fonction des stades du développement sexuel, femelle, l'étude des variations pondérales du tube digestif et de ses différentes parties montre (fig. $2 B$ ), (tableau II); - une décroissance pondérale rapide du tube digestif et de ses différentes parties

I. Il s'agit de la radioactivité par unité de volume (ml) des deux liquides de boisson.

2. Cf. discussion. 


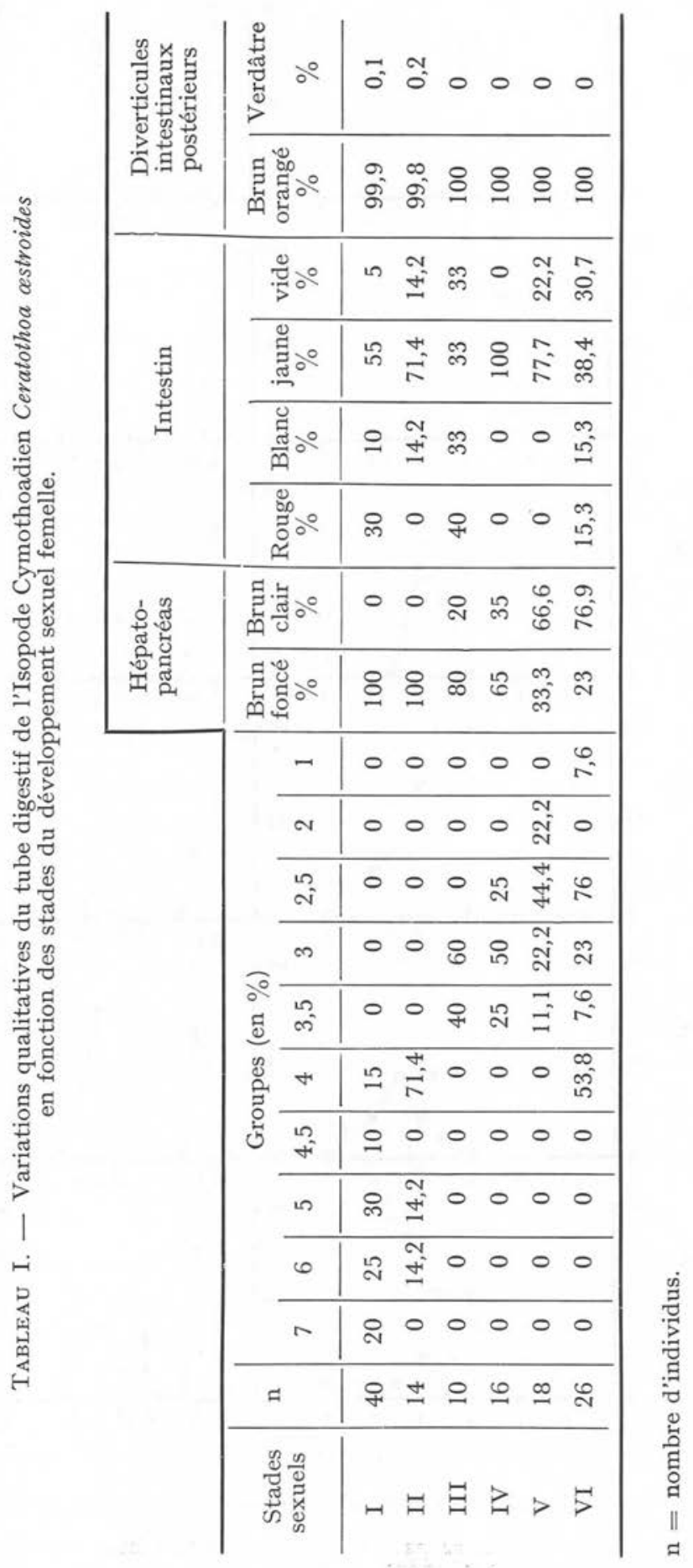



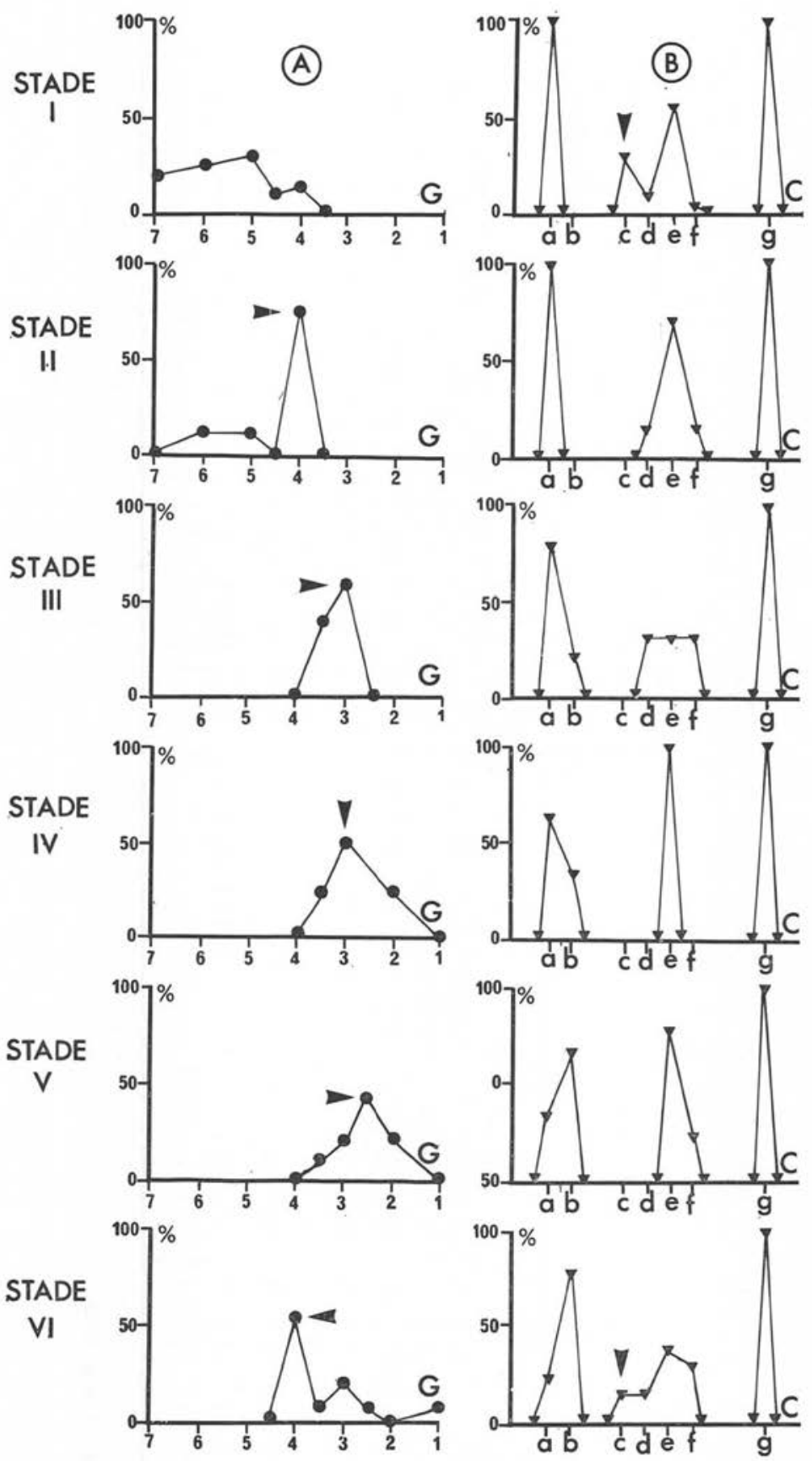

FIG. I. - Variations en fonction des différents stades du développement sexuel de Ceratothoa cestroides femelle :

A) Des phases de réplétion de l'hépatopancréas (abscisse : G : groupes ; ordonnée : pourcentage des individus).

B) De la coloration des différentes parties du tube digestif (abscisse : $\mathrm{C}=$ colorations ; ordonnées : pourcentage des individus).

a) hépatopancréas brun foncé; $b=$ hépatopancréas brun clair ; c) intestin rempli d'une substance rouge; $\mathrm{d}=$ intestin rempli d'une substance blanchâtre ; e) intestin rempli d'une substance jaune clair ; $f=$ intestin vide ; $g=$ diverticules intestinaux postérieurs brun orangé. 
(A)

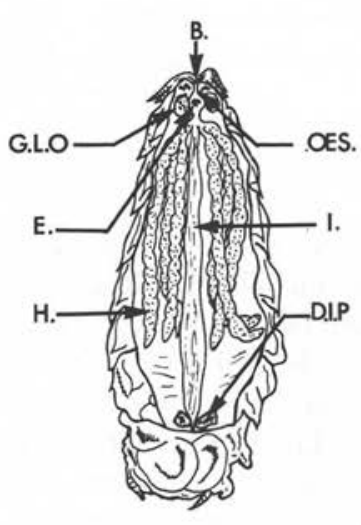

(B)

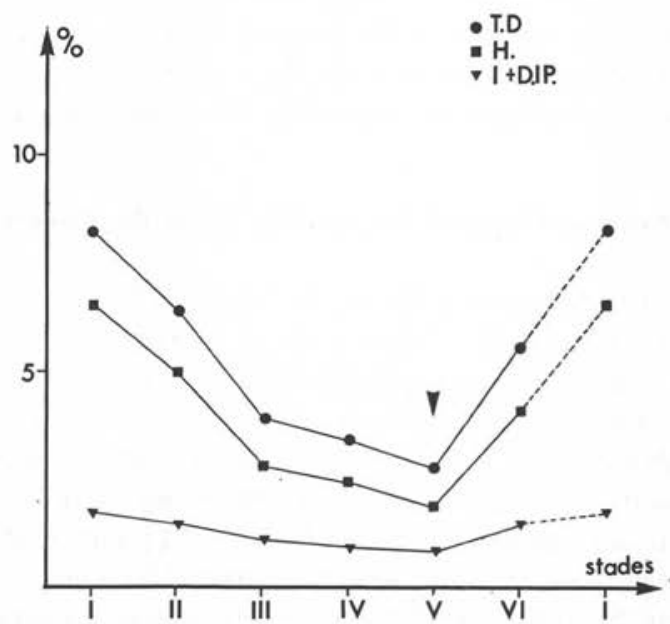

FIG. 2. - A) Représentation schématique du tube digestif de Ceratothoa cestroides femelle;

B) Variations pondérales du tube digestif complet et de ses différentes parties, en fonction des stades du développement sexuel.

(abscisse : les stades sexuels; ordonnée : poids de tube digestif complet, de l'hépatopancréas, de l'intestin et des diverticules intestinaux postérieurs (exprimé en pourcentage du poids frais de l'animal).

B : Bouche; D.I.P. : diverticules intestinaux postérieurs ; E : Estomac ; G.L.O. : Glandes latéro-œesophagiennes; H : Hépatopancréas ; I : Intestin; OES : Esophage ; T.D. : Tube digestif.

(hépatopancréas, intestin avec diverticules intestinaux postérieurs) du stade I au stade III ;

- une décroissance pondérale plus lente et beaucoup plus progressive du stade III au stade $\mathrm{V}$ inclus.

TABLEAU II. - Variations pondérales* du tube digestif, hépatopancréas, intestin plus diverticules intestinaux postérieurs et longueurs de l'hépatopancréas** en fonction des stades de développement sexuel femelle de Ceratothoa cestroides.

\begin{tabular}{|c|c|c|c|c|c|c|}
\hline Stades sexuels $q$ & I & II & III & IV & V & VI \\
\hline $\begin{array}{l}\text { Tube digestif } \\
\text { Hépatopancréas }\end{array}$ & $\begin{array}{l}8,26 \\
6,55\end{array}$ & $\begin{array}{l}6,49 \\
5,04\end{array}$ & $\begin{array}{l}3,92 \\
2,81\end{array}$ & $\begin{array}{l}3,41 \\
2,49\end{array}$ & $\begin{array}{l}2,78 \\
1,88\end{array}$ & $\begin{array}{l}5,59 \\
4,10\end{array}$ \\
\hline $\begin{array}{l}\text { Intestin + diverticules intestinaux } \\
\text { postérieurs } \\
\text { Longueurs de l'hépatopancréas }\end{array}$ & 1,71 & 1,45 & $\begin{array}{l}1,11 \\
3,5\end{array}$ & 0,92 & $\begin{array}{l}0,90 \\
2,5\end{array}$ & $\begin{array}{l}1,48 \\
3,5\end{array}$ \\
\hline
\end{tabular}

* Les variations pondérales sont exprimées en \% du poids frais de l'animal.

** Les chiffres indiqués représentent le segment péréial atteint par l'extrémité postérieure de l'hépatopancréas. 
- une croissance pondérale nette du tube digestif du stade V au stade VI.

Les décroissances sont très vraisemblablement consécutives à l'utilisation des substances ingérées et des réserves hépatopancréatiques lors de la vitellogenèse et du développement " intramarsupial ». Par contre, une reprise de l'alimentation après la mise bas explique les augmentations pondérales observées du stade V au stade VI.

\section{Mécanismes hypothétiques de prise de nourriture}

Des mesures utilisant du $51 \mathrm{Cr}$ EDTA, réalisées sur des Ceratothoa cestroides en stade III adaptés à l'eau de mer ou à l'eau de mer diluée au demi (Thuet et Romestand, à paraître), nous ont permis de noter que le taux de boisson est plus élevé en eau de mer diluée (valeur de l'ordre de $5,3 \mu \mathrm{l} / \mathrm{h} / \mathrm{g})$ qu'en eau de mer $(0,73 \mu \mathrm{l} / \mathrm{h} / \mathrm{g})$. Ce résultat diffère de tous ceux jusqu'à présent connus chez les Crustacés, en particulier non parasites, qui devenant hyperosmotiques en milieu dilué, réduisent souvent massivement leur taux de boisson (Maetz, 1974 ; Thuet, 1979-1980). Il est probable que cette particularité est liée à l'hématophagie. En effet, ces parasites s'alimentent exclusivement de sang de poisson dont la pression osmotique est de l'ordre de 370 milliosmoles, voisine de celle de l'eau de mer diluée au demi (550 m-osmoles).

Afin de vérifier les résultats précédents, nous avons évalué le taux de boisson.

- Une première série d'expériences utilisant des individus adaptés à l'eau de mer au demi, dont le céphalon est baigné par de l'eau de mer (lot I avec pression osmotique égale à $1100 \mathrm{~m}$ osmoles) ou de l'eau de mer diluée (lot II : avec pression osmotique égale à $370 \mathrm{~m}$ osmoles) montre que l'ingestion du liquide est environ 4 fois plus importante chez les individus du lot II. Ce n'est donc pas la concentration du liquide baignant les pléopodes qui est responsable de l'augmentation importante de l'absorption du milieu en eau de mer diluée au $1 / 2$ mais bien la concentration du liquide au contact du céphalon.

- Une deuxième série d'expériences a été réalisée de telle manière que les conditions expérimentales soient aussi proches que possible des conditions naturelles : les animaux ont la partie postérieure (lames pléopodiales) immergée dans de l'eau de mer. Dans ce cas, l'absorption du liquide de boisson est encore environ 4 fois plus importante pour les 18 animaux dont le céphalon a été recouvert par de l'eau de mer diluée (pression osmotique de $370 \mathrm{~m}$ osmoles) que pour les 21 animaux dont le céphalon est recouvert d'eau de mer (1 $100 \mathrm{~m}$ osmoles).

Pour quantifier ces résultats, nous avons reconduit l'expérience précédente en ajoutant uniquement aux différents liquides de boisson une solution d'EDTA $51 \mathrm{Cr}$ et en établissant la durée de l'absorption à 4 heures.

Les résultats consignés dans le tableau III montrent qu'en EM le taux de boisson est plus faible $(0,31 \mu \mathrm{l} / \mathrm{h} / \mathrm{g})$ qu'en EM diluée $(1,18 \mu \mathrm{l} / \mathrm{h} / \mathrm{g})$. Ils confirment donc ceux obtenus par la méthode d'appréciation semi-quantitative. Il est à signaler que ces taux de boisson sont inférieurs à ceux trouvés après immersion totale d'individus en EM normale $(0,7 \mu \mathrm{l} / \mathrm{h} / \mathrm{g})$ et diluée $(5 / 10)(5,2 \mu \mathrm{l} / \mathrm{h} / \mathrm{g})$ (Thuet et Romestand, sous presse). 
TABLEAU III. - Taux de boisson EDTA ${ }^{51} \mathrm{Cr}$ chez Ceratothoa cestroides en EM normale et diluée.

Eau de mer : 1100 mosmol. Eau de mer : 398 mosmol.

Taux de boissons

$(\mu \mathrm{l} / \mathrm{h} / \mathrm{g})$ poids frais

$0,31 \pm 0,10$

$1,18 \pm 0,70$

Nombre d'individus

Comparaison statistique : ces deux valeurs diffèrent significativement au seuil de $5 \%$ (analyse de variance).

L'ingestion semble donc dépendre de la pression osmotique du milieu baignant la cavité buccale. Il est vraisemblable que les récepteurs éventuels sensibles aux variations de la pression osmotique se trouvent dans la partie antérieure de l'animal baignée par le liquide de boisson coloré et radioactif.

\section{Conclusions - discussion}

De cette étude relative à certains mécanismes nutritionnels chez l'Isopode Cymothoidae $C$. cestroides femelle, nous pouvons retenir que : les variations qualitatives (réplétion de l'hépatopancréas, coloration des différents organes) et les variations quantitatives (variations pondérales du tube digestif) montrent que l'ingestion de sang s'effectue au stade I (avant la vitellogenèse), éventuellement au stade III (après la ponte) et au stade VI (après la mise bas). Ces stades sont caractérisés pas la présence fugace d'une partie du sang de l'hôte dans l'intestin et par l'aspect (couleur et longueur) de l'hépatopancréas. Après un repas sanguin, l'intestin d'abord gonflé de sang va progressivement expulser son contenu, grâce à des mouvements peristaltiques, dans les six caecums hépatopancréatiques (Fig. 2B). Ce phénomène semble assez rapide puisque des mesures de radioactivité effectuées au moment de la dissection sur les caecums et l'intestin montrent que les premiers ont une activité nettement plus importante.

Des stades I et II au stade V inclus, interviennent la digestion, l'absorption des substances résultant de la dégradation du sang (hémoglobine = hématéine, hématine et fer) et l'utilisation des réserves hépatopancréatiques. Au cours des trois premiers stades du développement, les différentes substances ingérées et mises en réserve sont rapidement utilisées, ce qui se traduit par une décroissance pondérale des différentes parties du tube digestif et surtout de l'hépatopancréas.

Par la suite, stades IV et V, le développement se poursuit, ainsi que l'utilisation des substances de réserves mais le dépôt de vitellus ayant eu lieu, la décroissance pondérale du tube digestif est beaucoup plus lente.

Les phénomènes d'excrétion probablement liés à la présence dans l'intestin d'un liquide blanc laiteux ou d'un liquide jaunâtre n'ont pu pour l'instant être élucidés ; toutefois nous avons pu noter que l'apparition de la substance blanchâtre dans 
l'intestin, au cours des stades qui suivent l'ingestion de sang, est vraisemblablement à relier à l'état de réplétion du tube digestif.

Comme les pranizes de Gnathiidae (Isopodes à parasitisme protélien, hématophages), les Cymothoidae ont des processus d'alimentation qui ne sont pas continus mais cycliques, au cours desquels se succèdent alternativement des périodes de succion de sang (stades I et VI) de poisson et des périodes de digestion et d'absorption (stades II à V) au niveau des caecums entériques. Ce résultat a d'ailleurs été pressenti par Trilles en 1968. Pour les Cymothoadiens femelles, ces phénomènes sont étroitement liés aux stades du développement sexuel et au cycle d'intermue. Ainsi, à la différence des stades larvaires des Gnathiidae où les processus de préparation à la mue sont liés à l'évolution digestive, la mue coïncidant avec la fin de la digestion du " repas sanguin " (Juchault-Stoll, 1963), chez les Cymothoidae la mue se réalise immédiatement après l'ingestion de sang (stades II-III), au tout début de la digestion ; elle est indispensable pour que l'évolution sexuelle se poursuive ; la femelle va ainsi acquérir les cinq paires d'oostégites constituant le marsupium à l'intérieur duquel les œufs vont se développer. L'évolution sexuelle s'effectue du stade III au stade V en utilisant les réserves produites par l'absorption des différentes substances résultant de la digestion sanguine. Au stade V, les réserves sont presque totalement épuisées et la libération des pulli a lieu. En stade VI, une nouvelle ingestion de sang intervient, la mue se produit, la femelle perd alors ses lames incubatrices et un nouveau cycle sexuel recommence.

Chez les Gnathiidae, comme l'a montré Mouchet (1928), il semble exister deux types de digestion selon que l'hémolyse des érythrocytes absorbés par le parasite se produit immédiatement après le repas ou au contraire plus tardivement. Dans le premier cas, il se forme de la biliverdine (pigment verdâtre) alors que dans le second cas, ce pigment n'apparaît pas. Chez les Cymothoidae bien que l'hémolyse se produise assez rapidement dans l'intestin et l'hépatopancréas (Romestand, 1979), nous n'avons pratiquement jamais noté d'apparition de biliverdine. Il est toutefois intéressant de préciser que sur 124 individus femelles expérimentées, deux présentaient des diverticules intestinaux postérieurs de coloration verdâtre. Ces derniers organes auraientils un rôle dans la transformation de l'hémoglobine en biliverdine dans des cas très particuliers à définir ? Ces cas trop exceptionnels n'ont pas pu pour l'instant être étudiés.

Enfin, chez les Cymothoadiens, le mécanisme de prise de nourriture pourrait être déclenché par le facteur pression osmotique ou par la composition ionique du sang de l'hôte. Divers auteurs ont d'ailleurs montré que chez les Insectes hématophages, la pression osmotique, la composition ionique ainsi que de nombreuses substances contenues dans le sang augmentent l'absorption (Langley, 1976). Étant donné que chez ces derniers, l'existence de chémorécepteurs, localisés au niveau des pièces buccales, a été mise en évidence, il est logique de penser à la suite de ces travaux et de nos résultats préliminaires, qu'il existe chez Ceratothoa cestroides un mécanisme semblable. Des expériences complémentaires, utilisant du sang total (globules et plasma) et diverses substances chimiques considérées comme phagostimulantes ou inhibitrices, seraient à envisager, afin de mieux préciser ce mécanisme. 


\section{BIBLIOGRAPHIE}

De Scalzi G. : Intorno ad un Isopode Cymothoidae parasita dello Smaris vulgaris. Boll. Pesca. Pisci. Idrobiol., I94I, XIII, 423-428.

FArN-MAurel M. : Contribution à l'histologie et à la caryologie de quelques Isopodes : spermiogenèse et infrastructure du spermatozoỉde des Oniscidés et des Cymothoidés. Acquisitions récentes sur les spermatogenèses atypiques : I-I87. Thèse d'État, Université Paris, série A $\mathrm{n}^{\circ} 4468, \mathrm{n}^{\circ}$ d'ordre 5316 , 1966 .

GabE M. : Données histochimiques sur les glandes exocrines céphalothroraciques d'Anilocra physodes Leach. Ann. Histochim., r960, 5, 55-63.

IDE M. : Le tube digestif des Édriophtalmes. Cellule, I893, 8, 97-204.

Juchault-Stoll C. : La mue en deux temps chez Paragnathia formica (Hesse) (Isopode Gnathiidae). Arch. Zool. Exp. Gén., 1963, 104, I-22.

LANGLEY P. A. : Initiation and regulation of ingestion by hematophagous Arthropods. J. Med. Ent., 1976, 13, 121-130.

Legrand J. J. : Contribution à l'étude expérimentale et statistique de la biologie d'Anilocra physodes. L. Arch. Zool. Exp. Gén., 1952, 89, 1-56.

Lombardo C. A. : Morfologia del dermascheletro del capodi Anilocra physodes L. (Crustacea, Isopoda, Cymothoidae). Cah. Biol. Mar., 1975, XVI, 301-316.

MAETZ J. : Aspects of adaptation to hypo-osmotic and hyper-osmotic environments. In : Biochemical and biophysical perspectives in marine biology. Vol. I : r-67. Ed. D. C. Malins and J. R. Sargent. Academic Press, London, New York. I974.

Monod Th. : Expédition Raumphius II (1975). Crustacés parasites, commensaux, etc. (Th. Monod et R. Serene, éd.) III. Crustacés Isopodes ( ${ }^{\text {re }}$ partie) (Corallanidae, Anilocridae, Cymothoidae). Bull. Mus. Hist. Nat., Paris, 1976, 391, 835-870.

Mouchet S. : Contribution à l'étude de la digestion chez les Gnathidae. Bull. Soc. Zool. Fr., I928, $53,442-452$,

Romestand B., Trilles J.-P. : Au sujet d'une substance à activité antithrombinique mise en évidence dans les glandes latéro-œesophagiennes de Meinertia œstroides (Risso, 1826) (Isopoda, Flabellifera, Cymothoidae); parasite de poissons). Z. Parasitenk., 1976, 50, 87-92.

Romestand B. : Étude écophysiologique des parasitoses à Cymothoadiens. Ann. Parasitol. Hum. Comp., 1979, s4, 423-448.

Schioedte J. C. : On the structure of the mouth in sucking Crustacea. Ann. Mag. Nat. Hist. Sér. 4 , I $868, i, \mathrm{I}-25$.

Thuer P. : L'ionorégulation et l'osmorégulation chez les Crustacés. 2. Aspects physiologiques Océanis, 1980 , 5, 769-797.

Thuet P., Romestand B. : Les transferts d'eau en fonction de la salinité du milieu chez deux Isopodes Cymothoidae : Meinertia astroides (Risso, I826) et Anilocra physodes (L., I758) (Parasites de Poissons marins). Arch. Internat. Physiol. Biochim. (à paraître).

TRILLES J.-P. : Recherches sur les Isopodes Cymothoidae des côtes françaises. Ire Thèse : bionomie, parasitisme, biologie générale, sexualité : I-793. $2^{\mathrm{e}}$ Thèse : systématique, faunistique : I-181. Thèse d'État, Université Montpellier, no CNRS, AO 2305. I968. 